\title{
Calcitonin's Fantastic Voyage: from Hormone to Marker of a Genetic Disorder*
}

Philippe Fragu

\section{Summary}

The story of thyroid calcitonin is an illuminating example of the voyage of hormone from a therapeutic tool for bone disease to a tumour marker to screen for subclinical forms of cancer. Identified as a new thyroid hormone implicated in calcium metabolism, its pharmacological action offered a new therapeutic tool for the management of bone disease. By measuring the circulating calcitonin, a range of values was obtained for oncologists because the evolution of a newly identified form of thyroid cancer medullary (MTC) - was poorly understood. Researchers' interest shifted from calcitonin physiological action to its use as tumour marker able to diagnose MTC, especially in genetically predisposed families. Then, oncologists and geneticists combined their efforts to identify genetic mutation(s) implicated in MTC, an example of the decoding that is demanded of contemporary laboratory-based medicine to recognise a pathological entity.

Keywords: endocrinology; genetic disorders; epistemology; laboratory medicine; cancer

\section{Introduction}

In her book on Fuller Albright (1900-1969), Le clinicien et le chercheur, Christiane Sinding emphasised the role of therapeutic or diagnostic tools that lead

* Acknowledgments:The study was funded by Inserm and a grant from the Fondation de France (Comité de Santé Publique et Pathologies Cancéreuses). The author would like to thank Professor François Delaporte (Université de Picardie Jules-Verne) for the critical and attentive rereading of the manuscript.

Philippe Fragu (Epistémologie et histoire des sciences biologiques et médicales, Université de Picardie Jules-Verne, Amiens), 58, boulevard Saint Marcel, F-75005 Paris (philippe.fragu @wanadoo.fr). 
to the creation of a nosological entity or the modification of how it is perceived by 20th-century laboratory-based medicine ${ }^{1}$. As of the 1920 s the progressive generalisation of biochemistry methods at the patient's bedside changed the configurations of various pathologies, which at that time were based only on the recognition of a specific anatomical lesion detected either histologically or radiologically. According to this new laboratory-based medicine, the physiological lesion appears first and requires specific decoding of the findings that leads to the demonstration of a given pathological entity. Furthermore, the biological sign can even indicate a potential disease, whose likelihood of developing is more-or-less elevated and depends on other factors. Since the 1960s the development of immunological methods, on the one hand, and molecular biology, on the other, has accentuated this impact of the biological sign on the diagnostic process. Laboratory-based medicine requires the continuous search for and development of biological diagnostic tools, leading to a continuous alignment between the normal and the pathological. To analyse this transformation of medicine, Peter Keating and Alberto Cambrosio introduced the concept of biomedical platforms defined as

material and discursive arrangements that act as the bench upon which conventions concerning the biological and normal are connected with conventions concerning the medical or pathological ${ }^{2}$.

To better understand this model of medicine, we have chosen to examine the origin and development of a specific diagnostic tool - calcitonin - based on the last 50 years of the history of endocrinology. The technical aspect of this text is explained by its being based on a study of original bibliographical sources. I consider this approach essential because most journals referred to as secondary retained only those findings for which a consensus was reached. It is the role of the historian to weave the available threads of the recitation, which, in the current setting of scientific publications, are not cited.

At the end of the 1950s endocrinology changed course. The broad endocrinological functions had already been put in place and most hormones described since 1900 had been identified chemically. Ever more precise biochemical techniques were able to measure the different organic and inorganic components in blood and extracellular fluids. Regulatory pathways were proposed that linked the variations of these components to different hormonal secretions. The theoretical model that received the most attention was the

1 Sinding 1991.

2 Keating/Cambrosio 2003, 332. Jean-Paul Gaudillière (2002) described the major changes in medical and biological practices which led to L'invention de la biomédecine in France. 
concept of feedback control proposed by the cyberneticist Norbert Wiener (1894-1964). According to that model, the information transmitted to the control mechanism(s) of a system could differentiate between a desired situation and the existing one; the response mechanism would then be put into motion to reduce the difference between the two. This kind of servomechanism reasoning was applied for the first time in 1949 to the couple formed by the thyroid and pituitary glands ${ }^{3}$. It opened the way for the study of the mechanisms of transmission of biological information 10 years before its application to genetic regulation ${ }^{4}$.

From the 1960s onwards, the studies that led to the identification of a new thyroid hormone - calcitonin, implicated in phosphate-calcium metabolism - were the result of endocrinologists' attempts to understand the mechanisms that maintained steady serum calcium levels. The discovery of calcitonin enabled them to devise a schema describing the regulatory pathway that included a role for this hypocalcaemia-inducing hormone and another, hypercalcaemia-inducing one, parathyroid hormone (PTH), which is secreted by the parathyroid gland. Determinations of the circulating concentrations of this new thyroid hormone provided a range of values for oncologists, enabling them to diagnose a certain form of thyroid cancer medullary thyroid cancer - and to follow its outcome (cure or progression) under treatment. As a result, calcitonin became a tumour marker of this heretofore poorly understood cancer and served as an indicator for the identification of the genetic mutation(s) in the familial forms of this cancer in the 1990s.

Based on this example, it is possible to understand the rules that were imposed on researchers to restrict and define the problems raised by their observations, and that they deemed worthy of investigation ${ }^{5}$. Oncologists took charge of the physiologists' calcitonin, which became a marker of a genetically transmitted disorder and guided their diagnostic and therapeutic strategies. The geneticists used calcitonin to select the families to participate in the search for molecular genetic lesions based on sera and tissues collected by the clinicians treating these patients. In short, this is a road map of how a diagnostic tool was constructed.

3 Wiener 1948; Hoskins 1949.

4 Fox Keller 2002.

5 Fleck 1979. 


\section{Calcitonin: a new thyroid hormone for calcium metabolism}

In 1950 the study of calcium metabolism, especially its bone component, was reinvigorated by the introduction of the new methods derived from biophysics. Electron microscopy, X-ray diffraction studies on bone tissues and autohistoradiography provided a better understanding of how new bone is formed and becomes a dynamic, perpetually renewed tissue. The use of radioactive calcium in conjunction with appropriate mathematical models enabled the quantification of this metabolism and showed that, in the young animal, the renewed blood calcium level per minute is around $100 \%$, meaning that total circulating calcium could be renewed every minute. Fuller Albright's investigations on hyperparathyroidism stimulated those on the role of the parathyroid glands, whose secretion product had been isolated in the $1920 \mathrm{~s}^{6}$. Implicated in calcium metabolism, their ablation in humans induced fatal hypocalcaemia, whereas their benign or malignant hypertrophy led to hypercalcaemia. Their secretion product - PTH - was obtained in a pure form in 1959; it is a polypeptide composed of 84 amino acids.

In 1955 the American physiologist Franklin McLean proposed a model of a feedback mechanism to maintain the circulating calcium level ${ }^{7}$. By adapting its secretion to the blood calcium level, the parathyroid gland plays the role of thermostat. When the calcium level is low, PTH secretion is increased, and calcium is released from bone to correct the deficit. In the case of calcium overload, PTH secretion is decreased, followed by the diminished release of calcium from the bone compartment. Experimental proof was only indirect and relied on the histological modifications observed in the parathyroid gland or bone, but the dynamics of these changes did not seem to be able to account fully for the stability of calcium levels in normal subjects. It was thus necessary to consider other actions of the hormone, for example: a direct effect on the chemical exchange between blood calcium and free bone calcium, and the digestive mechanisms of calcium adsorption or renal excretion of calcium. A major role in the control of blood calcium levels was proposed for PTH, but there was no indication of its mode of action so far ${ }^{8}$.

6 Albright/Reifenstein Jr. 1948; Li 1992.

7 McLean 1958. McLean was Professor of Medicine at the University of Chicago and played a crucial role in defining the task of full-time physicians to develop clinical investigation. See Kolher 1982, 241f.

8 Rasmussen 1961. 


\section{Demonstration of calcitonin}

Experimental models were developed that attempted to reproduce the conditions of hypercalcaemia, obtained by the slow infusion of calcium, or hypocalcaemia, provoked by the administration of ethylenediaminetetraacetic acid (EDTA), which forms a complex with circulating calcium, rendering the latter non-ionic and unusable. In 1962 the group headed by the Canadian physiologist Harold Copp devised a complex experimental procedure that enabled, on the one hand, to modulate the blood calcium level in the general circulation and, on the other hand, to make possible the study of calcium-concentration changes in blood samples drawn just after ablation of the thyroid-parathyroid, whose function was maintained by extracorporeal circulation. Then, the entry into action of PTH was recorded by successively alternating phases of hyper- and hypocalcaemia. Statistical analyses of the variations of the local calcium levels demonstrated that, after calcium overload, the blood calcium concentration was more rapidly normalised in the normal dog than the parathyroidectomised dog. Copp's group advanced the hypothesis of a second hormone with a rapid calcium-lowering action and provided solid arguments as to its parathyroid origin. Called calcitonin, this new parathyroid hormone broadened the concept of calcium feedback proposed by McLean, based on the opposite actions of the two hormones: PTH to raise and calcitonin to lower the blood calcium concentration'.

These results stimulated interest early in 1963 but were also the object of certain criticisms ${ }^{10}$. The first remarked that Copp et al. used an unpublished, but evidently extremely precise, method for the measurement of calcium levels, because the variation reported for duplicate samples was $0.05 \mathrm{mg} \%$, unlike the technique known at that time. Similarly, the Canadian group's protocol for preparing the parathyroid extract was not known, but the end product had a calcium-lowering activity (calcitonin), whereas industrial extracts were endowed exclusively with calcium-increasing activity (PTH). Finally, the extracts prepared with parathyroid glands from other species (rat, guinea pig) had no effect on blood calcium. Nevertheless, in September 1963, a group of London chemists directed by Iain MacIntyre confirmed the Canadians' observations. Their experimental protocol included a system of ionexchange resins that varied the calcium concentration in the blood afferent to the parathyroid glands, whereas the calcium concentration in the efferent blood was determined using a spectrophotometric method that had been

9 Copp et al. 1962.

10 Tashjian/Munson 1963. 
reported in 1961 by the same group. These researchers immediately suggested the possible existence of this hormone in other species, while emphasising that the canine model did not allow the absolute separation of parathyroid and thyroid glands ${ }^{11}$.

The debate on this hormone was launched anew by pharmacologists from Harvard University directed by Paul Munson. Unlike the physiologists, who usually used dogs in their experiments, pharmacologists conducted most of their tests on rats. The American group showed that the destruction of rat parathyroids by hot-wire cauterisation generated a more brutal drop in the blood calcium level than surgical ablation. This exaggerated calciumlowering action was attributed to thyroid lesions caused by the cautery and could be linked to the release from the thyroid of a calcium-lowering factor, analogous to that described previously; it was proposed calling it thyrocalcitonin. The latter team even devised a test to measure this hormone: after chemical preparation of a thyroid extract, its activity could be detected by its calcium-lowering effect in rat blood ${ }^{12}$. Does thyroid calcitonin differ from parathyroid calcitonin? That is the question researchers examining phosphate-calcium metabolism asked.

One of the difficulties of these physiological studies lies in the fact that, in numerous animal species, it is impossible to achieve perfusion of the parathyroid glands that is completely independent of the thyroid vascularisation, especially in dogs. To establish the exact site of secretion of this new hormone, an animal model had to be found that would enable unequivocal distinction between thyroid and parathyroid vascularisation. Diametrically opposed findings were obtained according to the animal model chosen. Copp's team repeated their physiological experiments on sheep, whose superior parathyroid glands are completely separated from the thyroid, and confirmed the strictly parathyroid origin of this secretion ${ }^{13}$. At the same time, MacIntyre's group demonstrated the thyroid origin in goats, which have two external parathyroid glands, whose vascularisation is completely independent of that supplying the thyroid, while the two internal parathyroids cannot be dissociated from the thyroid circulation ${ }^{14}$. Their demonstration provided biochemical proof: the parathyroid extract had no calcium-lowering activity, while the thyroid preparation was endowed with such activity. The thyroid extracts of numerous other species also possessed this calcium-lowering capacity, and several groups were trying to extract and purify the chemical

11 Kumar/Foster/MacIntyre 1963.

12 Hirsch/Gauthier/Munson 1963; Hirsch/Voeckel/Munson 1964.

13 Copp/Henze 1964.

14 Foster et al. 1964. 
component responsible. In 1965 thyrocalcitonin was isolated: it is a polypeptide ${ }^{15}$. It was finally demonstrated in the human thyroid by the French group headed by the biophysicist Gérard Milhaud and Munson's team ${ }^{16}$. The chemists' arguments carried the day and parathyroid calcitonin was considered an artefact.

\section{Cellular origin of calcitonin secretion}

The discovery of calcitonin's thyroid origin led to the reexamination of the histological structure of the thyroid gland. Organised around a central cavity to form the thyroid follicle, thyrocytes are responsible for the synthesis of iodinated thyroid hormones - thyroxine and triiodothyronines according to a pathway firmly established by the studies of the French biochemist Jean Roche (1901-1992) ${ }^{17}$. Thyrocytes, the predominant cells forming the thyroid gland, are stimulated by hypophyseal thyroid-stimulating hormone (TSH). However, hypophysectomy, which leads to thyrocyte regression and diminished thyroid hormone synthesis, had no effect on the calcitonin activity of thyroid tissue ${ }^{18}$. The physiology of another cell component of thyroid - the light cell - was then revisited.

In 1876 the English histologist E. D. Barber had described, in the dog, this second population of thyroid cells, much fewer in number but identifiable by their lower affinity for stains used to reveal histological structures and from which they draw their name, light cells of the thyroid ${ }^{19}$. Always located between thyroid follicles, light-cell existence remained controversial: an embryonic remnant for some, a sign of glandular hyperactivity in young animals for others, they were often considered artefacts associated with the orientation of the plane of the section. In 1932 José Nonidez applied the methods of argyrophil (silver impregnation) staining to study the innervation of the thyroid gland ${ }^{20}$. He observed that the light cells of the thyroid are selectively impregnated by silver nitrate. Their principal characteristic is the presence of numerous granules. The more common use of electron microscopes, at the end of the 1950s, enabled better differentiation between thyrocytes and light cells, whose cytoplasmic granules have very specific char-

15 Gudmundsson/MacIntyre/Soliman 1966; Tenenhouse/Arnaud/Rasmussen 1965.

16 Milhaud et al. 1965; Aliapoulios/Voelkel/Munson 1966.

17 Fragu 2003.

18 Milhaud/Moukhtar 1965.

19 Barber 1876.

20 Nonidez 1932. 
acteristics: binding of L-dopa and the presence of enzymes like cholinesterase or dehydrogenase ${ }^{21}$. At the beginning of the 1960s these light cells were thought to be either the product of thyrocyte degradation or cells with unknown endocrine potential ${ }^{22}$.

During a symposium on the parathyroid gland held in Chicago in 1964, a special session was devoted to the parathyroid blood-calcium-lowering factor ${ }^{23}$. MacIntyre's group provided new arguments in favour of its thyroid origin by combining the chemical methods of tissue extraction and those of cellular localisation, particularly those developed by the London histochemistry group directed by A. G. Everson Pearse ${ }^{24}$. The idea was to follow the ultrastructural and enzymatic modifications induced in light cells and thyrocytes by calcium overloading or depletion. They demonstrated that the glycerophosphate dehydrogenase content of light cells decreased after calcium overloading and observed the disappearance of cytoplasmic granules resembling those of secretion granules. These changes were not observed in thyrocytes. In 1967 Bussolati and Pearse used an immunofluorescence assay to localise thyrocalcitonin in light cells by means of its reactivity with an antithyrocalcitonin antibody ${ }^{25}$.

As a result, thyroid light cells became $\mathrm{C}$ (calcitonin) cells and it was important to identify their embryological origin. During the third week of embryonic development the thyroid body appears as a medial epithelial proliferation of the floor of the pharynx of endodermal origin, while the lateral part of the floor of the pharynx gives rise to the ultimobranchial body. By the time the thyroid reaches its final position on the anterior face of the trachea, the ultimobranchial body has been incorporated into the thyroid. In 1937 Melvin Godwin had hypothesised that light cells might be of ultimobranchial origin $^{26}$. In 1967 Pearse's group provided the first proof of the ultimobranchial origin of $\mathrm{C}$ cells, by following their successive localisations during embryogenesis, made possible by their histochemical properties. At the same time Copp's group isolated large quantities of calcitonin from the ultimobranchial bodies of fish and chickens ${ }^{27}$. Calcitonin became the hormone of the ultimobranchial body, whose cells migrated during embryogenesis into the thyroid or parathyroids, depending on the species, making it the macroscopic origin of the hormone's secretion.

21 Rasmussen 1997.

22 Young/Leblond 1964.

23 Gaillard/Talmage/Budy 1965.

24 Pearse 1953.

25 Foster/MacIntyre/Pearse 1964; Bussolati/Pearse 1967.

26 Godwin 1937.

27 Copp/Cockroft/Kueh 1967. 
The techniques devised to detect calcitonin, during the early 1960s, were those of classical physiology, which, by means of an astute experimental protocol, managed to detect a substance having an action on a constituent of the internal medium - calcium. The choices of the dog by the Canadian physiologists and the rat by the American pharmacologists clearly explain the discrepancies observed between their experimental findings, because dog parathyroids do contain $\mathrm{C}$ cells and calcitonin, whereas the principle source in other mammals is the thyroid, from which biochemical methods were able to determine the structure of the new hormone. At the end of the 1960s the physiological mysteries evoked by calcitonin were in part solved. Its pharmacological destiny appeared to be linked to its action on bone, where, by inhibiting bone catabolism, calcitonin offered a new therapeutic approach to the management of osteoporosis. The latter led pharmaceutical companies to commit large amounts of money to bring it to market and advertise its usefulness as a drug ${ }^{28}$. However, the search for a pathology specific to $\mathrm{C}$ cells was unsuccessful up to 1968. It was the hypotheses formulated as to the origin of an atypical cancer of the thyroid body - medullary thyroid cancer (MTC) - that shifted the interest in calcitonin to that of a specific marker of malignant transformation of $\mathrm{C}$ cells. A new diagnostic tool - the bloodcalcitonin assay - was forged and then put to use by other researchers oncologists - especially in the clinical context.

\section{Calcitonin: the tumour marker of MTC}

The concept of tumour markers appeared during the 1960s. They are proteins released into the circulation by a tumour and their monitoring allows the diagnosis of the tumour and surveillance of its outcome. The first tumour markers were identified for colon cancer (carcinoembryonic antigen) and primary cancer of the liver (alpha-fetoprotein); they correspond to the derepression of embryonic antigen synthesis, a process linked to the dedifferentiation of tumour cells. In addition, the ectopic production of a pituitary hormone (adrenocorticotrophic hormone, which stimulates the adrenal gland) by a non-endocrine tumour (cancer of the lung) was definitively established. In 1968 it was demonstrated that MTC and its metastases produce calcitonin in enormous quantities, which are released into the circulation, opening the way to the definition of a new tumour marker. The demonstration of this pathology merits being explained in detail.

28 Symposium on Thyrocalcitonin 1967; 1992. 
During the 1950s the histological classification of thyroid cancers distinguished between differentiated and undifferentiated cancers, and linked differentiation to survival rates ${ }^{29}$. Differentiated cancers are often able to bind radioactive iodine and may have a good prognosis, sometimes several decades, unlike undifferentiated thyroid cancers. The latter are unable to bind radioactive iodine, which puts them beyond the reach of available therapy; they may kill their hosts within a few weeks. In 1959 the thyroid-surgery group of Cleveland, whose pathologist was John B. Hazard, published a detailed description of a new type of thyroid cancer based on 21 cases $^{30}$. This tumour was a relatively well-encapsulated single nodule, composed of a homogeneous proliferation of small, round or fusiform, cytologically poorly differentiated cells. They were dispersed in islets or bands with no vesicular or papillary differentiation. The stroma, which was abundant and fibrous, was scattered in hyaline sheets that were selectively stained by reagents reacting with amyloid. The course was often chronic; 12 patients survived 6 to 27 years after the initial thyroid surgery, while 11 patients died (only 6 of cancers) 4 to 27 years after thyroidectomy. This cancer's principal characteristic was its intermediate prognosis between those of differentiated and undifferentiated malignancies. It was called medullary thyroid cancer.

This newly identified entity was rapidly confirmed by the publication, in 1961, of a retrospective series of 57 patients who had undergone surgery at the Mayo Clinic in Rochester between 1926 and $1955^{31}$. In 1965 the International Congress on the Thyroid Gland paid tribute to the existence of this new entity by devoting a special session to it, during which two new series of patients were described, for a total of 100 new patients. The largest cohort was that followed by I. Doniach's London group which also specialised in the experimental study of thyroid tumours in animals ${ }^{32}$.

However, the histological origin of MTC remained an enigma. Although its prognostic characteristics make it resemble differentiated cancers, it is unable to bind radioactive iodine. Its undifferentiated histological appearance is that of a tumour with a low mitotic index, unlike highly mitotic undifferentiated cancers. It was difficult to explain the development of these three types of thyroid cancers from a single thyrocyte, unless MTC was taken as an exception to the prevailing dogma that associated poor differentiation

29 Fragu 2000.

30 Hazard/Hawk/Crile Jr. 1959.

31 Woolner et al. 1961. The Mayo clinic is the main centre in US endocrinology field.

32 Williams/ Brown/Doniach 1966. 
with cell proliferation. In addition, autohistoradiography studies showed that radioactive iodine was never localised in amyloid deposits, which seemed to have no link with classical thyroid metabolism ${ }^{33}$.

The anatomical-clinical studies of Dillwyn Williams, the pathologist of Doniach's group, concurred with those of MacIntyre's chemists, whose research on calcitonin focused on thyroid C cells, notably in collaboration with Pearse's histochemistry group. Although these cells were difficult to identify in humans, in the rat they were the subject of numerous studies and were the most frequent origin of thyroid tumours, even though they do not possess all the characteristics of their human counterparts, especially their lack of amyloid. Proceeding by deduction, Williams was the first to suggest that MTC might be derived from thyroid $\mathrm{C}$ cells ${ }^{34}$. To him, this new concept appeared to be the only way to reconcile the good prognosis of these tumours, on the one hand, and their undifferentiated appearance, on the other. Originating from thyroid $\mathrm{C}$ cells, these tumours can never undergo papillary or follicular differentiation, which are exclusive properties of thyrocytes. The fundamental question remained whether or not these tumours secrete calcitonin.

\section{MTC, a calcitonin-secreting tumour}

During the first six months of 1968 MTC provided the decisive proof of the pathological secretion of calcitonin by C cells ${ }^{35}$. On January 25th, the American endocrinologist Edwin Astwood (1909-1976), acting as the rapporteur, presented to the National Academy of Sciences a case described by two Boston physicians, Kenneth Melvin and Armen Tashjian. Their female patient had a familial form of MCT associated with bilateral pheochromocytomas and a hepatic metastasis. The investigations conducted attempted to elucidate the mechanism(s) responsible for her low blood calcium. A liver biopsy showed the lesion to be a metastasis of her MTC, whose calcium-lowering activity was measured by the authors; it was 1,000 times higher than those found to date in human thyroids. In addition, calcitonin activity was elevated in her plasma, thereby suggesting that MTC is characterised by increased synthesis of calcitonin and its secretion into the circulation. Ten days later (5th February), the Academy of Sciences of Paris published a note, fruit of the collaboration of Milhaud's group and that of the oncologist, Maurice Tubiana. They described two patients with MTC in whom calcium-

33 Ljungberg 1966.

34 Williams 1966.

35 Melvin/Tashjian Jr. 1968; Milhaud et al. 1968; Meyer/Abdel-Bari 1968; Cunliffe et al. 1968. 
lowering activity was studied in blood and their thyroid lesions, and were compared to those of two patients with differentiated (papillary) cancers of the thyroid. No thyrocalcitonin activity was detected in either the tumours or metastatic lymph nodes of the two papillary carcinoma patients. In contrast, the two MTC (tumours and metastatic lymph nodes) exhibited marked thyrocalcitonin activity, which was 100 -fold higher than that found in normal thyroid tissue. Determined for only one of the two MTC patients, blood also exhibited intense calcium-lowering activity before surgery that disappeared thereafter. All these authors suggested that their findings supported Williams's hypothesis, that C cells were the origin of MTC.

Two other publications confirmed those observations, the first by a group of American pathologists, who described the ultrastructural characteristics of MTC. This tumour was composed of cells rich in secretion granules, whose appearance was identical to that of rat light cells. Given the high calcitonin content of the tumour examined, they concluded that these secretion granules were the site of calcitonin synthesis and storage. The second article reported the results of MacIntyre's and Williams's groups. The patient in question, again a woman, had MTC associated with dysmorphism resembling that of Marfan's syndrome (a skeletal and connective tissue disorder transmitted by autosomal dominant inheritance) and neurofibromatosis lesions. The patient's tumour and plasma were rich in calcium-lowering activity that was eluted from a Sephadex-50 ion-exchange column in the same fraction as pig calcitonin. Those researchers were the first to insist that calcitonin variations during treatment could be a diagnostic tool that should enable the detection of relapse.

In 1969 several communications confirmed the presence of $\mathrm{C}$ cells in the normal human thyroid with in situ detection of calcitonin. In MTC malignant transformation confers on $\mathrm{C}$ cells the ability to synthesise very large quantities of calcitonin, which is then released into the circulation, the only place where its calcium-lowering activity can be detected in humans. All these findings imposed changing calcitonin's status from hormone to tumour marker.

\section{Radioimmunoassay of calcitonin}

The calcitonin biological assay methods used until this time were based on lowering the blood calcium level in the rat. They took a long time and their sensitivity was insufficient to guarantee the reliability of the results and the successive monitoring of these concentrations, thereby rendering them poorly compatible with the clinical detection of medullary thyroid cancer and 
follow-up of its outcome. Therefore, the first step consisted of developing a radioimmunoassay (RIA) to measure circulating calcitonin.

The methods used to determine hormone concentrations in blood were completely transformed by the introduction of the radioimmunology principle by Rosalyn Yalow (1921-) and Salomon Berson at the beginning of the $1960 \mathrm{~s}^{36}$. Once a purified hormone has been obtained, it is possible to prepare a specific antibody directed against it and hence susceptible to bind to the hormone to form an antigen-antibody complex, and thus measure its blood level. As of 1969 the London and American groups applied this method to determine the calcitonin concentration ${ }^{37}$.

Even though calcitonin is made up of only 32 amino acids, it rapidly became apparent that it possesses very strong species specificity and that a human calcitonin RIA requires reagents of human origin. In addition, the antigenic sites exposed on the circulating forms of calcitonin varied widely, thereby rendering inconsistent their interaction with a standard antibody. The norms established for one laboratory were difficult to compare to those of another. Finally, the sensitivity of the RIA was low, with concentrations below $100 \mathrm{ng} / \mathrm{ml}$ being undetectable. Under these conditions circulating calcitonin was never detected in the normal subject and elevated values were observed for individuals with medullary thyroid cancer.

This lack of sensitivity, which is a real obstacle for physiological studies, also represented a stumbling block to the monitoring of the evolution of a medullary thyroid cancer, for which the objective is to detect the smallest possible tumours with very low calcitonin secretion at the very beginning of their progression or of their local relapse or metastatic spread. It was thus necessary to optimise the detection threshold by means of a hormonestimulation test, as routinely practised in endocrinology. These tests were based on the measurement of the plasma-calcitonin concentration during slow perfusion of calcium. Since 1974 the latter was replaced by an injection of pentagastrin, which contains the carboxyl-terminal tetrapeptide sequence of gastrin, known to physiologically induce calcitonin secretion during the digestive absorption of calcium ${ }^{38}$. These investigations were negative in healthy individuals but were presumably always positive when MTC was present.

The first series of patients with MTC showed that plasma-calcitonin concentrations were always elevated before any treatment; they declined after surgery and became undetectable in some patients, whose life prognoses were

36 Yalow/Berson 1960.

37 Clark/Boyd/Byfield/Foster 1969; Deftos 1971; Tashjian Jr. et al. 1970.

38 Cooper et al. 1971. 
the best. In addition, the variation of its level during monitoring enabled the detection of metastases before they became clinically manifest, especially intra-abdominal lymph-node metastases, which could not be visualised radiographically at that time ${ }^{39}$. By using staged catheterisation, enabling blood samples to be drawn at different levels in the body, the gradient of plasma calcitonin concentrations could be evaluated, thus representing the triumph of a biological sign over radiological images. As the biological sign detected a disease that was not clinically manifest, it was also used for the detection of familial forms when they appeared to be one of the main features of medullary thyroid cancer. Oncologists joined forces with geneticists to explain medullary thyroid cancer in terms of genetic lesion(s).

\section{Calcitonin: a guide for genetic studies}

Since the early 1950s research on syndromes associating multiple endocrine pathologies, especially neoplastic ones, has become a major field of investigation. The objective was to discover latent forms of the pathology by establishing the family tree or pedigree of the disease. In 1961 John Sipple described a female patient who had died of an adrenal medullar gland tumour - a pheochromocytoma - and in whom a malignant thyroid body tumour was discovered at autopsy ${ }^{40}$. Reexamining the reports on 537 pheochromocytomas that had been published to date, Sipple noted that a second malignant tumour had been associated in 22 of them; the most common was cancer of the thyroid, with seven reported cases representing $32 \%$ of associated tumours. He thought this frequency exceptionally high in light of the rarity of thyroid cancer in the general population (8/10 000 according to autopsy reports). Up until 1965, the several case reports on patients with this combined disease (called Sipple's syndrome) that had been published emphasised the presence of associated cutaneous lesions of neurofibromatosis.

Medullary thyroid cancer, high frequency among multiple endocrine neoplasias

In 1965, upon the publication of two new associations of pheochromocytoma and thyroid cancer, Williams compiled the characteristics of 17 patients with

39 Goltzman et al. 1974.

40 Sipple 1961. 
this pathological association published since $1932^{41}$. Their pheochromocytomas were usually bilateral, whereas they were unilateral in $90 \%$ of the patients in the general population. For six patients, this pathology was familial, having occurred in parents and children. Reexamination of histological sections of associated thyroid tumours led to the diagnosis of MTC in 11 patients; in addition, thyroid cancer was familial for four of them. At the same time, the results of two genealogical studies, one American and the other Swedish, suggested that the association of pheochromocytoma and MTC might be the expression of the same genetic disorder transmitted by autosomal dominant inheritance ${ }^{42}$.

For Williams, the pheochromocytoma-thyroid association was similar to that linking pheochromocytoma to the cutaneous lesions of neurofibromatosis. Pheochromocytomas are derived from adrenal medullary cells, whose embryological origin is the neural crest, which gives rise to the nervous system. Therefore, it was not surprising to see these familial forms associated with bilateral pheochromocytomas and the neurocutaneous syndromes, as Williams concluded in his analysis of published cases ${ }^{43}$. All these arguments led to the postulate that the neural crest could also be the embryological origin of the C cells responsible for MTC. However, the neural embryological origin of the $\mathrm{C}$ cells remained to be verified to explain the coexistence of pheochromocytomas.

\section{Medullary thyroid cancer, a tumour derived from cells originating} in embryonic neural crest

At the beginning of the 1970s calcitonin-containing cells in the ultimobranchial body of birds and mammalian thyroid glands were reported to share several of their characteristics with other cells secreting polypeptides and distributed throughout the organism (pituitary, pancreas and along the digestive tube), and they were regrouped by Pearse under the heading of the amine-content precursor uptake and decarboxylase (APUD) system ${ }^{44}$. Their properties include high levels of amine precursors, such as dihydroxyphenylalanine (DOPA) or 5-hydroxytryptophan (5-HT), and enzymes, for example alpha-glycerophosphate dehydrogenase, esterase and cholesterase. The essential feature of these cells is their ability to incorporate substances

41 Williams 1965.

42 Schimke/Hartmann 1965; Ljungberg/Cederquist/von Studnitz 1967.

43 Williams/Pollock 1966.

44 Pearse 1979. 
like dopa and 5-HT, decarboxylate them and subsequently produce biogenic amines that can be detected in tissue sections. In addition, electron microscopy revealed that these cells contained protein secretion granules. Carried along with the ultimobranchial tissue, nothing proves that they share the same origin. Couldn't it be a secondary migration of cells having their embryological origin in another embryonic layer, the neural crest for example? Using the method of heterospecific neural tube grafts between quail and chicken embryos, whose cells have distinct cytochemical characteristics, Le Douarin and Le Lièvre demonstrated that cells of neural origin containing intracytoplasmic granules typical of $\mathrm{C}$ cells colonised the outpocketing of the ultimobranchial body. Pearse and Polak confirmed this observation several months later ${ }^{45}$. Thus, the dual embryological roots of the thyroid underline the strong degree of interdependence of the endocrine and nervous systems, which are intricately linked by hormonal regulation and in the constitution of endocrine organs.

Because pheochromocytoma and MTC are derived from cells with the same embryological origin - the neural crest - a genetic defect of the stem cells might explain the occurrence, in the same patient, of these two pathologies. Hence, a search was initiated for this potential genetic anomaly in the siblings of these patients, and then their parents, their parents' siblings and their offspring, their grandparents, and so on. Could systematic determinations of blood calcitonin in members of families with MTC orient the diagnosis, like the measurement of urinary catecholamines (metabolites of adrenal medulla hormone) for the diagnosis of familial forms of pheochromocytoma? That is the question that oncologists and geneticists were asking at the beginning of the 1970s.

\section{Calcitonin: marker of familial forms of medullary thyroid cancer}

The answer was obtained in 1972, based on the results of systematic bloodcalcitonin determinations in the first familial inquiry, which included 89 blood relatives and three generations ${ }^{46}$. That analysis started by asking one of the family members living in Boston about the coexistence of thyroid cancer in one of his sisters and a cousin. Before the initiation of systematic biological screening, seven histologically proven MTCs had been observed, one associated with bilateral pheochromocytomas. In addition, this family of Swedish origin was related to the family that had been investigated five years earlier

45 Le Douarin/Le Lièvre 1970; Pearse/Polak 1971.

46 Melvin/Tashjian Jr./Miller 1972. 
by a group of Swedish physicians. All these patients had undergone a previous thyroid examination, which was negative for 88 of them. The only one with a morphological thyroid anomaly was found to have MTC after histological examination of the surgically excised gland. A complementary biological analysis examined blood samples, drawn before, during and after a calcium-stimulation test, and concomitantly collected urine specimens. Finally, the activity of a plasma enzyme, histaminase, which had been reported to be elevated in patients with MTC, was determined because it was also considered a potential marker of this malignancy ${ }^{47}$.

That investigation led to the demonstration, in 12 family members, of detectable plasma calcitonin concentrations that could be stimulated by calcium perfusion. All these patients, who were clinically asymptomatic, accepted to undergo preventive thyroidectomies; bilateral MTC were found at the junction of the upper and middle thirds of each lobe in all of them. Thus, the predictive value of the calcitonin concentration was demonstrated by a familial inquiry, while measurement of plasma histaminase activity proved less informative for the initial diagnosis, as well as for subsequent monitoring.

At the beginning of 1975, after the publication of the genealogical studies of two additional American families, it was agreed that there must be two forms of MTC: one sporadic, usually with unilateral lesions, and the other familial with bilateral involvement ${ }^{48}$. In practice, familial investigations were systematically undertaken in all the blood relatives of patients with this pathology. The aim was to identify calcitonin-secretion abnormalities, which enable the initiation of treatment for the underlying MTC before its metastatic spread, especially to lymph nodes. Calcitonin was not only a tumour marker but also the marker of an autosomal genetic disorder susceptible of undergoing malignant transformation.

\section{Calcitonin reveals $C$-cell hyperplasia}

It is in the study of the familial forms that the measurement of the circulating calcitonin concentration would prove heuristic, because it can diagnose the first step of malignant transformation - C-cell hyperplasia - allowing the formulation of a genetic theory of MTC.

Very rapidly a modification of the circulating calcitonin concentration and, especially, an amplified response during the hormone-stimulation test

47 Baylin et al. 1970.

48 Keiser 1973; Jackson/Tashjian Jr./Block 1973. 
led the Boston group to perform total thyroidectomies in patients with family histories of MTC. In 1973 they described three sisters who had this biological profile but no MTC was found during microscopy examination of the surgically resected tissues. The only anomaly identified was the presence of C-cell hyperplasia, homogeneously distributed at the junction of the upper and middle thirds of each thyroid lobe, the usual site of MTC, as opposed to their heterogeneous distribution in the normal thyroid gland. In addition, the calcitonin contents of these hyperplastic cells were highly elevated. Even though C-cell hyperplasia had already been described in surgically removed specimens from patients with hyperparathyroidism or hyperthyroidism, those authors advanced the hypothesis that, because of the genetic penetrance of MTC $(100 \%)$, this lesion precedes the appearance of MTC ${ }^{49}$.

This carcinogenesis in two stages - C-cell hyperplasia followed by malignant transformation - would be another example of the model being developed at the same time by Alfred Knudson of a particular form of ocular tumour - retinoblastoma ${ }^{50}$. When the latter appears early in childhood, the tumour is always bilateral, multifocal and of familial origin, unlike the late forms, which are unilateral, unifocal and sporadic. Knudson's so-called '2-hit' hypothesis stated that the first 'hit' in the development of a familial tumour occurs in the germline of a cancer-susceptibility gene and the second 'hit' is somatic, affecting the other allele of the same gene. Knudson's hypothesis also extended to encompass the development of sporadic tumours, with the two hits being somatic and affecting both alleles of the susceptibility gene. For familial MTC, C-cell hyperplasia would be the phenotypic expression of the genetic mutation, which requires a second somatic mutation to transform the cell bearing the first mutation into a cancer cell ${ }^{51}$. Although this theoretical model proved to be erroneous for MTC, it stimulated the search for cytogenetic and then molecular anomalies in the different familial forms of MTC because the first 'hit' can be detected.

At the beginning of the 1980s calcitonin enabled the establishment of a conceptual framework, in which research on familial forms of medullary thyroid cancer developed. The low frequency ( $5 \%$ of thyroid cancers, whose annual incidence of 6/100 000 inhabitants, affecting 2 men and 4 women) led to the formation of a multidisciplinary group to study these tumours. These investigations led to the identification of the genetic lesion of familial forms of medullary thyroid cancer: a mutation of the RET gene, located in the pericentromeric region of chromosome 10 , that codes for a membrane receptor

49 Wolfe et al. 1973; Aliapoulios/Rose 1970.

50 Knudson 1971.

51 Jackson et al. 1979. 
whose ligand is a protein implicated in the differentiation and migration of neuroectodermal cells ${ }^{52}$.

In France, the Group for the Study of Calcitonin-Expressing Tumours (GETC) was formed in 1984 by the group of Milhaud and gave itself several objectives: to set up a national registry; to establish a national protocol for the detection, monitoring and treatment; and to create a research network ${ }^{53}$. GETC, which has collected 2,700 patients with medullary thyroid cancer over 15 years, is an example of the construction of medical knowledge within the framework of contemporary laboratory-based medicine. It is responsible for managing case reports, serum and tissue banks, and histological slides, all elements to which new technologies are applied as soon as they are discovered, for example, the search for mutations of the RET oncogene ${ }^{54}$. GETC is an authentic illustration of new trends in laboratory-based medicine, in which specialists, who have no contact with the patient, construct the natural history of the disease piece by piece 'without' him/her ${ }^{55}$.

It is also a good example of probabilistic medicine, for which the curative intervention is modulated by the evolution of the disease itself, guided by calcitonin. At present, among the healthy members of a family at risk for the development of MTC, only those individuals with a genetic mutation in RET are systematically monitored with repeated assays of calcitonin; should it exceed $10 \mathrm{pg} / \mathrm{ml}$ during a pentagastrin-stimulation test, a total thyroidectomy is systematically performed, even in the absence of any clinical manifestation of thyroid cancer and regardless of the patient's age ${ }^{56}$. This strategy is meaningful only if the notion of a family history of MTC is known. It is more difficult to defend it for an isolated detection of calcitonin in the absence of any thyroid lesion that can be visualised. Indeed, no systematic study has been conducted on the detection of C-cell hyperplasia in the general population without family histories of MTC. We have reached the limits of this biologi-

52 Mulligan/Ponder 1995.

53 Calmettes 1984.

54 Sobol et al. 1989.

55 In France, this new biomedical approach was first developed by Jean Dausset (Nobel Prize 1980) who established in 1984 the Centre d'étude du polymorphisme humain (CEPH). To test the histocompatibility-gene (HLA) hypothesis in organ transplantation, it was necessary to constitute a large population in which HLA family-based linkage could be performed. In 1989, when polymerase chain reaction (PCR) profoundly transformed the practices and potential of molecular biology, the association of $C E P H$ and the Association Française contre les myopathies enlarged the purpose to all genes involved in hereditary diseases (Rabinow 1999). Another example of this avenue of new biomedical practices is furnished by the breast cancer genetics in which Gilbert Lenoir, in charge of GETC genetic studies, was engaged with the American physician Henry Lynch, who collected a large population of predisposed families in Nebraska (Cassier/Gaudillière 2000).

56 Nicolli-Sire et al. 1999. 
cal diagnostic tool, whose application is meaningless without reference to a family history.

\section{Conclusion}

The story of calcitonin is an illuminating example of the process developed within the laboratory-based medicine - that of a medical tool as a material support for the genesis of medical knowledge ${ }^{57}$.

At first, calcitonin was thought to be a hormone, whose action was the opposite of that of PTH. The basic method of identification was a bioassay demonstrating its effect on the blood calcium level. Then chemical methods were applied to purify calcitonin, determine its structure and prescribe it to treat clinical disorders of calcium metabolism. This has been the normal trajectory of a hormone since the discovery of the first scheme of endocrinology - that of the thyroid gland in $1892^{58}$. However, in humans, the physiological meaning of calcitonin has remained controversial. In a recent review, its discoverer, Philip Hirsch, wrote:

40 years after its discovery, it is time to recognize that the proposed functions (of calcitonin), if they exist at all, do not appear to be physiologically important and that mammalian calcitonin, although not yet vestigial, is likely to be in a gradual process of vestigialization. ${ }^{59}$

Regardless of the putative action of calcitonin, one of the main contributions of the first step of its path was to elucidate the physiology of thyroid C cells and their ultimobranchial origin, which opened a new avenue for understanding thyroid cancer.

Concomitantly, the effervescence of thyroid oncology constituted an incontestable element that stimulated studies on $\mathrm{C}$ cells and subsequently on calcitonin. The problem was to understand the cell from which MTC originated and the decisive factor was its abnormal production of calcitonin, the only human pathology in which this hormone is oversecreted without evident clinical repercussion. The focus on calcitonin shifted from fundamental endocrinology to clinical oncology leading to the development of a calcitonin RIA for diagnosis and therapeutic monitoring. The hormone became a tumour marker, the second step of its trip.

57 Clark/Fujimura 1992. More recently Sinding (2000) has analysed the fantastic 'voyage' of a molecule - glycosylated haemoglobin - as it passed successively from potential genetic marker to that of an abnormal protein synthesised in diabetics, to finally become a means of monitoring and managing diabetes.

58 Canguilhem 1989.

59 Hirsch/Baruch 2003. 
The coexistence of MTC with other endocrinological tumours - especially pheochromocytomas - in identified families led to the demonstration that these tumours were of the same embryonic origin: the neural crest. Furthermore, it appeared that calcitonin could even diagnose subclinical forms of MTC in predisposed familial forms. Then oncologists and geneticists worked together to identify the genetic mutation(s) responsible for the disease. By adopting this style of joint endeavour and creating a calcitonin research study group, the oncologists and geneticists contributed to the discovery and development of calcitonin as a diagnostic tool of a genetic disease. In this third step of its fantastic voyage, calcitonin has become the humoural marker used for genetic disease monitoring, which is defined by the calcitonin response to a stimulating agent.

In short, the story of thyroid calcitonin shows that its potential changed as different possibilities emerged from a therapeutic tool for bone disease to a tumour marker to screen for subclinical forms of cancer. It is an illuminating example of the decoding that is demanded of contemporary laboratory-based medicine to recognise a pathological entity. In the familial form of medullary thyroid carcinoma the biological sign detected by a diagnostic tool, the calcitonin RIA, replaced the clinical sign and the tissue lesion became a molecular one.

\section{References}

Albright,Fuller/E. C. Reifenstein Jr., The parathyroid glands and metabolic bone disease: selected studies (Baltimore 1948)

Aliapoulios, Menelaos A./Edward D. Voelkel/Paul L. Munson, "Assay of human thyroid glands for thyrocalcitonin activity", J. Clin. Endocrinol. 26 (1966) 897-901

- /Elizabeth H. Rose, "Specific localisation of calcitonin activity in human thyroid glands", Calcitonin 1969 (1970) 295-300

Barber, E. D., "Contributions to the minute anatomy of the thyroid gland of the dog", Proc. R. Soc. Lond. Ser. B 24 (1876) 240-241

Baylin, S. B. et al., "Elevated histaminase activity in medullary carcinoma of the thyroid gland", N. Engl. J. Med. 283 (1970) 1239-1244

Bussolati, G./A. G. E. Pearse, "Immunofluorescent localization of calcitonin in the 'C' cells of pig and dog thyroid", J. Endocrinol. 37 (1967) 205-209

Calmettes, Claude, «Création d'un groupe d'étude des tumeurs à calcitonine», Bull. Cancer (Paris) 71 (1984) 266-273

Canguilhem, Georges, «Pathologie et physiologie de la thyroïde au XIX ${ }^{\mathrm{e}}$ siècle», in: ibid., Etudes d'histoire et de philosophie des sciences (Paris 1989) 274-294

Cassier, Maurice/Jean-Paul Gaudillière, «Recherche, médecine et marché: la génétique du cancer du sein», Sciences sociales et santé 18 (2000) 29-51

Clark, Adele/Joan Fujimura (eds), The right tools for the job. At work in twentieth-century life sciences (Princeton 1992)

Clark, M. B./G. W. Boyd/P. G. Byfield/C. V. Foster, "A radioimmunoassay for human calcitonin M", Lancet ii (1969) 74-77

Cooper, Carry W. et al., “Thyrocalcitonin: stimulation of secretion by pentagastrin”, Science 172 (1971) 1238-1240 
Copp, D. Harold et al., "Evidence for calcitonin - a new hormone from the parathyroid that lowers blood calcium", Endocrinology 70 (1962) 638-649

- /K. G. Henze, "Parathyroid origin of calcitonin - evidence from perfusion of sheep glands", Endocrinology 75 (1964) 49-55

- /D. W. Cockroft/Yankoon Kueh, "Calcitonin from ultimobranchial glands of dogfish and chickens", Science 158 (1967) 924-925

Cunliffe, W. J. et al., "A calcitonin-secreting thyroid carcinoma", Lancet ii (1968) 63-66

Deftos, L. J., "Immunoassay for human calcitonin, I Method", Metabolism 20 (1971) 1122-1128

Fleck, Ludwig, Genesis and development of a scientific fact (Chicago 1979)

Foster, G. V./I. MacIntyre/A. G. E. Pearse, "Calcitonin production and the mitochondrion-rich cells of dog thyroid", Nature 203 (1964) 1029-1030

- et al., "Thyroid origin of calcitonin", Nature 202 (1964) 1303-1305

Fox Keller, Evelyn, Making Sense of Life (Cambridge 2002)

Fragu, Philippe, «Nosologie du cancer thyrö̈dien différencié: rôle des outils diagnostiques et thérapeutiques (1800-1950)», Bull. Cancer (Paris) 87 (2000) 145-154

- "How the field of endocrinology developed in France after World War II", Bull. Hist. Med. 77 (2003) 394-415

Gaillard, Pieter J./Roy V. Talmage/Ann M. Budy (eds), The Parathyroid Glands. Ultrastructure, secretion and function (Chicago 1965)

Gaudillière, Jean-Paul, Inventer la biomédecine, la France, l'Amérique et la production des savoirs du vivant (1945-1965) (Paris 2002)

Godwin, Melvin Crawford, "Complex IV in the dog with special emphasis on the relation of the ultimobranchial body to interfollicular cells in the post-natal thyroid gland", Am. J. Anat. 60 (1937) 299-340

Goltzman, D. et al., "Calcitonin as a tumor marker. Use of the radioimmunoassay for calcitonin in the postoperative evaluation of patients with medullary thyroid carcinoma", N. Engl. J. Med. 290 (1974) 1035-1039

Gudmundsson, T. V./I. MacIntyre/H. A. Soliman, "The isolation of thyrocalcitonin and a study of its effects in the rat", Proc. R. Soc. Lond. Ser. B 164 (1966) 460-477

Hazard, John B./William A. Hawk/George Crile, Jr., "Medullary (solid) carcinoma of the thyroid - a clinicopathologic entity", J. Clin. Endocrinol. 19 (1959) 152-161

Hirsch, Philip F./Geraldine H. Gauthier/Paul L. Munson, "Thyroid hypocalcemic principle and recurrent laryngeal nerve injury as factors affecting the response to parathyroidectomy in rats", Endocrinology 73 (1963) 244-252

- /Edward F. Voeckel/Paul L. Munson, "Thyrocalcitonin hypocalcemic hypophosphatemic principle of the thyroid gland", Science 145 (1964) 412-413

- /Hans Baruch, "Is calcitonin an important physiological substance?", Endocrine 21 (2003) 201-208

Hoskins, R. G, “The thyroid pituitary apparatus as a servo (feed-back) mechanism”, J. Clin. Endocrinol. 9 (1949)1429-1431

Jackson, Charles E./Armen H. Tashjian Jr./Melvin A. Block, "Detection of medullary thyroid cancer by calcitonin assay in families", Ann. Intern. Med. 78 (1973) 845-852

- et al., "The two-mutational-event theory in medullary thyroid cancer", Am. J. Hum. Genet. 31 (1979) 704-710

Keating, Peter/Alberto Cambrosio, Biomedical Platforms, Realigning the Normal and the Pathological in Late-Twentieth-Century Medicine (Cambridge 2003)

Keiser, Harry R., "Sipple's syndrome: medullary thyroid carcinoma, pheochromocytoma, and parathyroid disease", Ann. Intern. Med. 78 (1973) 561-579

Knudson Jr., Alfred G., "Mutation and cancer: statistical study of retinoblastoma", Proc. Natl. Acad. Sci. USA 68 (1971) 820-823

Kohler, Robert E., From Medical Chemistry to Biochemistry: the making of a biomedical discipline (Cambridge 1982)

Kumar, M. Ashwini/G. V. Foster/I. MacIntyre, "Further evidence for calcitonin. A rapid-acting hormone which lowers plasma-calcium", Lancet ii (1963) 480-482

Le Douarin, Nicole/Christiane Le Lièvre, «Démonstration de l'origine neurale des cellules à calcitonine du corps ultimobranchial chez l'embryon de poulet», C. R. Acad. Sci. Paris Sér. D 270 (1970) 2857-2860 
Li,Alison, "J.B.Collip,A. M. Hanson and the isolation of the parathyroid hormone, or endocrines and enterprise", J. Hist. Med. Allied Sci. 47 (1992) 405-438

Ljungberg, Otto, "Medullary carcinoma of the human thyroid gland. Autoradiographic localization of radioiodine", Acta Pathol. Microbiol. Scand. 68 (1966) 476-480

- /Ebbe Cederquist/Wilfried von Studnitz, "Medullary carcinoma and pheochromocytoma: a familial chromaffinomatosis", Br. Med. J. 1 (1967) 279-281

McLean, Franklin C., "The ultrastructure and function of bone", Science 127 (1958) 451-456

Melvin, Kenneth E. W./Armen H. Tashjian Jr., "The syndrome of excessive thyrocalcitonin produced by medullary carcinoma of the thyroid", Proc. Natl. Acad. Sci. USA 59 (1968) 1216-1222

- IArmen H.Tashjian Jr./Harry H. Miller, "Studies in familial (medullary) thyroid carcinoma", Recent Prog. Horm. Res. 28 (1972) 399-470

Meyer, John S./Wagih Abdel-Bari, "Granules and thyrocalcitonin-like activity in medullary carcinoma of the thyroid gland", N. Engl. J. Med. 278 (1968) 523-529

Milhaud, Gérard et al., «Existence et activité de la calcitonine chez l'homme», C. R. Acad. Sci. Paris 261 (1965) 4513-4516

- Mohsen S. Moukhtar, «Hypophysectomie et thyrocalcitonine», C. R. Acad. Sci. Paris Sér. D 260 (1965) 3179-3182

- et al., «Epithélioma de la thyroïde sécrétant de la thyrocalcitonine», C. R. Acad. Sci. Paris Sér. D 266 (1968) 608-610

Mulligan L. M./B. A. J. Ponder, "Genetic basis of endocrine disease: multiple endocrine neoplasia type 2", J. Clin. Endocrinol. Metab. 80 (1995) 1989-1995

Nicolli-Sire, P. et al., "Early or prophylactic thyroidectomy in MEN2/FMTC gene carriers: results in 71 thyroidectomized patients", Eur. J. Endocrinol. 141 (1999) 468-474

Nonidez, José F., "The origin of the 'parafollicular' cell, a second epithelial component of the thyroid gland of the dog", Am. J. Anat. 49 (1932) 479-505

Pearse, A. G. Everson, Histochemistry theoretical and applied (London 1953)

- J. M. Polak, "Cytochemical evidence for the neural crest origin of mammalian ultimobranchial C-cells", Histochemie 27 (1971) 96-102

- "The cytochemistry and ultrastructure of polypeptide hormone producing cells of the APUD series and the embryologic, physiologic and pathologic implications of the concept", J. Histochem. Cytochem. 17 (1979) 303-313

Rabinow, Paul, French DNA, Trouble in Purgatory (Chicago 1999)

Rasmussen, Howard, "Parathyroid hormone. Nature and mechanism of action", Am. J. Med. 30 (1961) 112-128

Rasmussen, Nicolas, Picture Control. The electron microscope and the transformation of biology in America, 1940-1960 (Stanford 1997)

Schimke, R. Neil/William H. Hartmann, "Familial amyloid-producing medullary thyroid carcinoma and pheochromocytoma.A distinct genetic entity", Ann. Intern. Med. 63 (1965) 1027-1039

Sinding, Christiane, Le clinicien et le chercheur. Des grandes maladies de carence à la médecine moléculaire (Paris 1991)

- «Une molécule espion pour les diabétologues, l'innovation entre science et morale», Sciences sociales et santé 18 (2000) 95-120

Sipple, John H., "The association of pheochromocytoma with carcinoma of the thyroid gland", Am. J. Med. 31 (1961) 163-166

Sobol, Hagay et al., "Screening for multiple endocrine neoplasia type 2a with DNA-polymorphism analysis", N. Engl. J. Med.321 (1989) 996-1001

"Symposium on Thyrocalcitonin", Am. J. Med. 43 (1967) 645-726

"Symposium celebrating the 30th anniversary of the discovery of calcitonin", Bone Miner. 16 (1992) 155-216

Tashjian Jr., Armen H./Paul L. Munson, "Chemical nature and properties of parathyroid hormones", J. Chron. Dis. 16 (1963) 269-281

- et al., "Immunoassay of human calcitonin. Clinical measurement, relation to serum calcium and studies in patients with medullary carcinoma", N. Engl. J. Med. 283 (1970) 890-895

Tenenhouse, Alan/Claude Arnaud/Howard Rasmussen, "The isolation and characterization of calcitonin", Proc. Natl. Acad. Sci. USA 53 (1965) 818-821 
Wiener, Norbert, Cybernetics or Control and Communication in the Animal and the Machine (Cambridge 1948)

Williams, E. D., "A review of 17 cases of carcinoma of the thyroid and pheochromocytoma", J. Clin. Pathol. 18 (1965) 288-292

- /C.L.Brown/I.Doniach,"Pathological and clinical findings in a series of 67 cases of medullary carcinoma of the thyroid", J. Clin. Pathol. 19 (1966) 103-113

- "Histogenesis of medullary carcinoma of the thyroid", J. Clin. Pathol. 19 (1966) 114-118

- /D. J. Pollock, "Multiple mucosal neuromas with endocrine tumors: a syndrome allied to von Recklinghausen's disease", J. Pathol. Bacteriol. 91 (1966) 71-80

Wolfe, Hubert J. et al., "C-cell hyperplasia preceding medullary thyroid carcinoma", N. Engl. J. Med. 289 (1973) 437-441

Woolner, Lewis B. et al.,"Classification and prognosis of thyroid carcinoma. A study of 855 cases observed in a thirty-year period", Am. J. Surg. 102 (1961) 354-387

Yalow, Rosalyn S./Solomon A. Berson, "Immunoassay of endogenous plasma insulin in man", J. Clin. Invest. 39 (1960) 1157-1175

Young, B. A./Charles P. Leblond, "The light cell as compared to the follicular cell in the thyroid gland of the rat", Endocrinology 73 (1964) 669-686 